\title{
VARIABILITY REGIONS FOR BOUNDED ANALYTIC FUNCTIONS WITH APPLICATIONS TO FAMILIES DEFINED BY SUBORDINATION
}

\author{
YUSUF ABU-MUHANNA AND THOMAS H. MACGREGOR
}

\begin{abstract}
We examine the set of points $\left(\varphi(\zeta), \varphi^{\prime}(\zeta), \ldots, \varphi^{(n)}(\zeta)\right)$ where $|\zeta|<1$ and $\varphi$ varies over the class of functions analytic in the open unit disk and is either (1) uniformly bounded or (2) subordinate to a given univalent function. In each case boundary points of the set correspond to unique functions associated with finite Blaschke products. This yields information about the form of solutions to extremal problems over the classes, including the problem
\end{abstract}

$$
\max \operatorname{Re} F\left(\varphi(\zeta), \varphi^{\prime}(\zeta), \ldots, \varphi^{(n)}(\zeta)\right)
$$

where $|\zeta|<1$ and $F$ is analytic.

1. Introduction. Let $\Delta=\{z:|z|<1\}$ and let $A$ denote the set of functions analytic in $\Delta$. Let $B$ denote the subset of $A$ consisting of functions $\varphi$ that satisfy $|\varphi(z)|<1$. If $|\zeta|<1$ then $B_{n}(\zeta)$ shall denote the $n$th region of variability of $B$ $(n=1,2, \ldots)$. The set $B_{n}(\zeta)$ consists of those points $\left(w_{1}, w_{2}, \ldots, w_{n}\right)$ in $\mathbf{C}^{n}$ for which there is a function $\varphi$ in $B$ so that $\varphi^{(k-1)}(\zeta)=w_{k}$ for $k=1,2, \ldots, n$ (where $\left.\varphi^{(0)}=\varphi\right)$.

We shall prove the following theorem about $B_{n}(\zeta)$.

TheOREM 1. $B_{n}(\zeta)$ is a compact, convex subset of $\mathbf{C}^{n}$. Each boundary point of $B_{n}(\zeta)$ corresponds to a unique function $\varphi$ in $B$. Such a function has the form

$$
\varphi(z)=x \prod_{k=1}^{m} \frac{z+\alpha_{k}}{1+\bar{\alpha}_{k} z}
$$

where

$$
|x|=1, \quad\left|\alpha_{k}\right|<1 \quad(k=1,2, \ldots, m) \text { and } m<-1 .
$$

The set of extreme points of $b_{n}(\zeta)$ is the boundary of $B_{n}(\zeta)$.

Any function $\varphi$ given by equation (1) where $|x|=1$ and $\left|\alpha_{k}\right|<1 \quad(k=$ $1,2, \ldots, m)$ shall be called a finite Blaschke product of degree $m$. The proof of Theorem 1 uses the corresponding fact about the $n$th coefficient region of $B$, which we denote by $B_{n}$. A point $\left(c_{0}, c_{1}, \ldots, c_{n-1}\right)$ belongs to $B_{n}$ if there is a function $\varphi$ in $B$ so that

$$
\varphi(z)=\sum_{k=0}^{\infty} d_{k} z^{k} \quad(|z|<1)
$$

Received by the editors April 2, 1979.

AMS (MOS) subject classifications (1970). Primary 30A40, 30A76.

Key words and phrases. Analytic function, bounded function, variability region, extreme point, coefficient region, finite Blaschke product, extremal problems, subordination, univalent function. 
with $d_{k}=c_{k}$ for $k=0,1, \ldots, n-1$. It is a classical result of J. Schur [9], [10] that each boundary point of $B_{n}$ corresponds to a unique function in $B$ and such a function is a finite Blaschke product of degree at most $n-1$.

Theorem 1 can be used to obtain facts about the set of solutions to various extremal problems over $B$. The following two theorems give extremal problems for which the general form of the solution set can be identified.

THEOREM 2. Suppose that $|\zeta|<1$ and the functional $J$ is defined on $A$ by

$$
J(\varphi)=\sum_{k=0}^{n-1} b_{k} \varphi^{(k)}(\zeta)
$$

where $\varphi \in A$ and $b_{k} \neq 0$ for some $k$. There is a unique function $\varphi_{0}$ in $B$ so that

$$
\operatorname{Re} J\left(\varphi_{0}\right)=\max \{\operatorname{Re} J(\varphi): \varphi \in B\} .
$$

Moreover, $\varphi_{0}$ is a finite Blaschke product of degree at most $n-1$.

Conversely, if $\varphi_{0}$ is a finite Blaschke product of degree at most $n-1$ then there is a function $J$ defined on $A$ and having the form of equation (4) so that $\varphi_{0}$ is the only function in $B$ satisfying equation (5).

THEOREM 3. Suppose that $|\zeta|<1$ and the functional $J$ is defined on $B$ by

$$
J(\varphi)=F\left(\varphi(\zeta), \varphi^{\prime}(\zeta), \ldots, \varphi^{(n-1)}(\zeta)\right)
$$

where $F$ is a nonconstant function analytic on the interior of $B_{n}(\zeta)$ and continuous on $B_{n}(\zeta)$. The only functions $\varphi_{0}$ in $B$ for which $\operatorname{Re} J\left(\varphi_{0}\right)=\max \{\operatorname{Re} J(\varphi): \varphi \in B\}$ are finite Blaschke products of degree at most $n-1$.

We also prove two theorems similar to Theorems 1 and 3 for the set of functions in $A$ that are subordinate to a given univalent function. In particular, Theorem 5 provides a generalization of the following known result (see [4], [6], [7], [8]).

TheOREM A. Let $P$ denote the subset of $A$ consisting of functions $p$ so that $p(0)=1$ and $\operatorname{Re} p(z)>0$ for $|z|<1$. Suppose that $|\zeta|<1$ and the functional $J$ is defined on $P$ by

$$
J(p)=F\left(p(\zeta), p^{\prime}(\zeta), \ldots, p^{(n-1)}(\zeta)\right)
$$

where $F$ is a function analytic on the nth region of variability of $P$. The only functions $p_{0}$ in $P$ for which $\operatorname{Re} J\left(p_{0}\right)=\max \{\operatorname{Re} J(p): p \in P\}$ have the form

$$
p_{0}(z)=\sum_{k=1}^{n} \lambda_{k} \frac{1+x_{k} z}{1-x_{k} z}
$$

where

$$
\sum_{k=1}^{n} \lambda_{k}=1, \quad \lambda_{k} \geqslant 0 \text { and }\left|x_{k}\right|=1 \quad(k=1,2, \ldots, n) .
$$

Theorem $\mathrm{A}$ was proved in the cited references by using variational techniques. Our approach is quite different and clarifies the relations on which such results depend. We identify the form of functions associated with boundary points of the variability region and then apply the maximum principle for harmonic functions. 
The boundary points are described by examining the nature of the map between variability regions induced from the map between the class and $B$. Because of the information given by Schur's algorithm the form of extremal functions becomes clear, including the exact number of parameters. The technique of solving general extremal problems depending on coefficients by examining properties of the coefficient regions was recently used by A. Atzmon in [1] and by P. C. Cochrane and the second author in [3].

2. Proofs of theorems about $B$.

Proof of TheOREM 1. $B_{n}(\zeta)$ is compact since $B$ is compact in the topology of uniform convergence on compact subsets of $\Delta$. Likewise, $B_{n}(\zeta)$ is convex since $B$ is convex.

If $|\zeta|<1$ then the correspondence $\varphi \rightarrow \psi$ defined by

$$
\psi(z)=\varphi((z+\zeta) /(1+\bar{\zeta} z))
$$

provides a one-to-one mapping from $B$ onto $B$. This is a consequence of the fact that $w=(z+\zeta) /(1+\bar{\zeta} z)$ gives a one-to-one map of $\Delta$ onto $\Delta$ and thus equation (10) may be solved for $\varphi$ in terms of $\psi$ through the inverse linear transformation. If we let

$$
\psi(z)=\sum_{n=0}^{\infty} c_{n} z^{n}
$$

and

$$
\gamma_{n}=\varphi^{(n)}(\zeta) \quad(n=0,1, \ldots)
$$

then $c_{0}=\gamma_{0}$ and by differentiating equation (10) we may express $c_{n}$ in terms of $\gamma_{n}$ for $n \geq 1$. We find that

$$
n c_{n}=\sum_{k=1}^{n} \alpha_{n k} \gamma_{k} \quad(n=1,2, \ldots)
$$

where $\alpha_{n k}$ depend on $\zeta$ and, in particular,

$$
\alpha_{n n}=\left(1-|\zeta|^{2}\right)^{n}
$$

Suppose that a correspondence $\left(\gamma_{0}, \gamma_{1}, \ldots, \gamma_{n-1}\right) \rightarrow\left(c_{0}, c_{1}, \ldots, c_{n-1}\right)$ from $\mathbf{C}^{n}$ to $\mathbf{C}^{n}$ is defined by $c_{0}=\gamma_{0}$ and $c_{m}=\sum_{k=1}^{m-1} \beta_{m k} \gamma_{k}(m=1,2, \ldots, n-1)$ where $\beta_{m k}$ are given complex numbers. If $\beta_{m m} \neq 0$ for $m=1,2, \ldots, n-1$, then this correspondence is a homeomorphism of $\mathbf{C}^{n}$ onto $\mathbf{C}^{n}$. This is a consequence of the condition $\beta_{m m} \neq 0$, which permits the successive solution of $\gamma_{0}, \gamma_{1}, \ldots, \gamma_{n-1}$ in terms of $c_{0}, c_{1}, \ldots, c_{n-1}$ (the matrix defining the linear map is nonsingular since it is lower triangular and each diagonal element is not zero). This implies that the mapping between $B_{n}(\zeta)$ and $B_{n}$ induced through equation (10) is a homeomorphism $\left(\beta_{m k}=\alpha_{m k} / m\right.$ and from equation (14) $\left.\alpha_{m m} \neq 0\right)$. Therefore, boundary points of $B_{n}(\zeta)$ correspond to boundary points of $B_{n}$.

Suppose that $p$ is a boundary point of $B_{n}(\zeta)$ and $\varphi$ is any function in $B$ associated with $p$. Then the function $\psi$ defined by equation (10) produces a boundary point $q$ of $B_{n}$. Because of Schur's result $q$ uniquely associates with $\psi$ and 
$\psi$ is a finite Blaschke product of degree at most $n-1$. Since the mapping from $B$ onto $B$ given by equation (10) is one-to-one this implies that $\varphi$ is the only function associated with $p$. Moreover, $\varphi$ must be a finite Blaschke product of degree at most $n-1$. This is a consequence of the characterization of finite Blaschke products through the properties $\varphi \in A, \varphi$ is continuous in $\bar{\Delta}$ and $|\varphi(z)|=1$ for $|z|=1$ (see [2, pp. 12-13]). If $\psi$ has these properties then so does $\varphi$. Also, $\varphi$ has the same degree as $\psi$ since each function maps $\Delta$ onto $\Delta$ with the same multiplicity.

The proof of the statement about the extreme points of $B_{n}(\zeta)$ is the same as that given for the extreme points of $B_{n}$ (see [3, Lemma 2]). The argument depends on the facts that each boundary point of $B_{n}(\zeta)$ is associated with a unique function $\varphi$ which is a finite Blaschke product and that if $\varphi, \psi$ and $\omega$ are any three such products for which $\varphi=t \psi+(1-t) \omega$ with $0<t<1$ then $\psi=\omega=\varphi$.

Proof of Theorem 2. This argument is the same as that given for the corresponding extremal problem for coefficients (see [3, Theorem 1]). The main idea is to note that if $M=\max \{\operatorname{Re} J(\varphi): \varphi \in B\}$ then the inequality $\operatorname{Re} J(\varphi)<M$ defines a support plane to $B_{n}(\zeta)$. This plane can contain only one point in $B_{n}(\zeta)$ since each boundary point of $B_{n}(\zeta)$ is an extreme point and proper convex combinations of two finite Blaschke products are not a Blaschke product. The converse statement holds by introducing the functional defining the support plane to $B_{n}(\zeta)$ at the boundary point associated with $\varphi_{0}$.

Proof of TheORem 3. The properties of the function $F$ imply that $\operatorname{Re} F\left(w_{1}, w_{2}, \ldots, w_{n}\right)$ achieves its maximum only when $\left(w_{1}, w_{2}, \ldots, w_{n}\right)$ is a boundary point of $B_{n}(\zeta)$. By Theorem 1 such points are (uniquely) associated with finite Blaschke products of degree at most $n-1$.

3. Families defined by subordination. Throughout this section we assume that $f \in A$ and $f$ is univalent in $\Delta$. Let $S(f)$ denote the set of functions $g$ that are subordinate to $f$ in $\Delta$. This means that $g \in A, g(0)=f(0)$ and $g(\Delta) \subset f(\Delta)$. These conditions on $g$ are equivalent to the existence of a function $\omega$ satisfying $\omega \in A$, $\omega(0)=(0)$ and $|\omega(z)|<1$ for $|z|<1$ so that $g(z)=f(\omega(z))$. Since $\omega(z)=z \varphi(z)$ where $\varphi \in B$, the set $S(f)$ is the same as the collection of functions $g$ given by

$$
g(z)=f(z \varphi(z))
$$

where $\varphi$ varies over $B$. Equation (15) defines a one-to-one correspondence between $B$ and $S(f)$ since whenever $f$ is nonconstant the condition $f\left(\omega_{1}\right)=f\left(\omega_{2}\right)$ implies $\omega_{1}=\omega_{2}$ for pairs of functions $\omega_{1}, \omega_{2}$ in $B$ (through local properties of analytic functions).

Let $S_{n}(\zeta)$ denote the $n$th region of variability of $S(f)$. Thus, if $|\zeta|<1$ then $\left(w_{1}, w_{2}, \ldots, w_{n}\right) \in S_{n}(\zeta)$ if there is a function $g$ in $S(f)$ so that $g^{(k-1)}(\zeta)=w_{k}$ for $k=1,2, \ldots, n$.

THEOREM 4. $S_{n}(\zeta)$ is a compact subset of $\mathbf{C}^{n}$. If $\zeta \neq 0$ then each boundary point of $S_{n}(\zeta)$ uniquely corresponds to a function $g$ in $S(f)$ and $g$ has the form $g(z)=f(z \varphi(z))$ where $\varphi$ is a finite Blaschke product of degree at most $n-1$. If $\zeta=0$ and $n>2$ the same conclusion holds where the Blaschke product has degree at most $n-2$. 
Proof. $S_{n}(\zeta)$ is compact since the family $S(f)$ is compact.

Suppose that $g \in S(f)$ and $g$ corresponds to $\varphi$ in $B$ through equation (15). We first consider the case $\zeta \neq 0$. Equation (15) implies that $g(\zeta)=f(\zeta \varphi(\zeta)$ ). By differentiating that equation we find that

$$
g^{(k)}(\zeta)=G_{k}\left(\varphi(\zeta), \varphi^{\prime}(\zeta), \ldots, \varphi^{(k-1)}(\zeta)\right)+\alpha_{k} \varphi^{(k)}(\zeta)
$$

for $k=1,2, \ldots$, where $G_{k}$ is an analytic function on $\bar{\Delta} \times \mathbf{C}^{k-1}$ and $\alpha_{k}=$ $\zeta f^{\prime}(\zeta \varphi(\zeta))$. Since $\zeta \neq 0$ and $f$ is univalent in $\Delta$ it follows that $\alpha_{k} \neq 0$. This implies that the correspondence $\left(z_{1}, z_{2}, \ldots, z_{n}\right) \rightarrow\left(w_{1}, w_{2}, \ldots, w_{n}\right)$ from $\bar{\Delta} \times \mathbf{C}^{n-1}$ into $\mathbf{C}^{n}$ defined by

$$
w_{1}=f\left(\zeta z_{1}\right), \quad w_{k}=G_{k}\left(z_{1}, z_{2}, \ldots, z_{k-1}\right)+\alpha_{k} z_{k} \quad(k=1,2, \ldots, n-1),
$$

is a homeomorphism. The condition $\alpha_{k} \neq 0$ and $\zeta \neq 0$ make it possible to solve this system for $z_{1}, z_{2}, \ldots, z_{n-1}$ successively in terms of $w_{1}, w_{2}, \ldots, w_{n-1}$. This proves that the relation (15) induces a homeomorphism of $B_{n}(\zeta)$ onto $S_{n}(\zeta)$. In particular, each boundary point of $B_{n}(\zeta)$ corresponds to a unique boundary point of $S_{n}(\zeta)$ through this homeomorphism.

An appeal to Theorem 1 completes the proof of this theorem in the case $\zeta \neq 0$.

We now prove the statement when $\zeta=0$. If we let $f(z)=\sum_{n=0}^{\infty} a_{n} z^{n}, g(z)=$ $\sum_{n=0}^{\infty} b_{n} z^{n}$ and $\varphi(z)=\sum_{n=0}^{\infty} c_{n} z^{n}$, then equation (15) implies that $b_{0}=a_{0}$ and $b_{n}=H_{n}\left(c_{0}, c_{1}, \ldots, c_{n-2}\right)+a_{1} c_{n-1}$ for $n=1,2, \ldots$ where $H_{n}$ is a polynomial. Since $a_{1} \neq 0$ the correspondence from $\mathrm{C}^{n-1}$ to $\mathrm{C}^{n-1}$ defined by

$$
b_{m}=H_{m}\left(c_{0}, c_{1}, \ldots, c_{m-2}\right)+a_{1} c_{m-1} \quad(m=1,2, \ldots, n-1)
$$

is a homeomorphism. Therefore, the mapping (15) induces a homeomorphism between $B_{n-2}$ and the set of points $\left(b_{1}, b_{2}, \ldots, b_{n-1}\right)=\left(g^{\prime}(0), 2 ! g^{\prime \prime}(0), \ldots\right.$, $\left.(n-1) ! g^{(n-1)}(0)\right)$. Since $b_{0}=a_{0}$ for all functions $g$ in $S(f)$ we have a homeomorphism between $B_{n-2}$ and $S_{n}(0)$. In particular, each boundary point of $S_{n}(0)$ corresponds to a boundary point of $B_{n-2}$. Again, an appeal to Schur's result implies that each boundary point of $S_{n}(0)$ corresponds to a unique function $g$ in $S(f)$ where equation (15) holds and $\varphi$ is a finite Blaschke product of degree at most $n-2$.

THEOREM 5. Suppose that $|\zeta|<1$ and $F$ is a nonconstant function analytic on the interior of $S_{n}(\zeta)$ and continuous on $S_{n}(\zeta)$. If $\zeta \neq 0$ then the only functions in $S(f)$ that maximize $\operatorname{Re} F\left(g(\zeta), g^{\prime}(\zeta), \ldots, g^{(n-1)}(\zeta)\right)$ over $S(f)$ have the form $g(z)=f(z \varphi(z))$ where $\varphi$ is a finite Blaschke product of degree at most $n-1$. If $\zeta=0$ and $n>2$ the same conclusion holds where the Blaschke product has degree at most $n-2$.

Proof. This is an immediate consequence of Theorem 4 and the fact that $\operatorname{Re} F\left(w_{1}, w_{2}, \ldots, w_{n}\right)$ achieves its maximum over $S_{n}(\zeta)$ only when $\left(w_{1}, w_{2}, \ldots, w_{n}\right)$ is on the boundary of $S_{n}(\zeta)$.

Remarks. 1. Theorem 5 includes Theorem $A$ as a special case since $P$ consists of the functions subordinate to the univalent function $f(z)=(1+z) /(1-z)$. Also, when $\varphi$ is a finite Blaschke product of degree at most $n-1$ the function $g(z)=f(z \varphi(z))$ has the form given by equations (8) and (9) (see [3, p. 83]).

There have been various applications of Theorem $A$ in the last several years. We 
anticipate useful applications of Theorem 5 for families related to subordination with specific univalent functions $f$.

2. The arguments given in this paper provide a means of determining the variability regions. For example, the inequalities $\left|c_{0}\right|<1,\left|c_{1}\right|+\left|c_{0}\right|^{2}<1$ and $\left|\left(1-\left|c_{0}\right|^{2}\right) c_{2}+\bar{c}_{0} c_{1}^{2}\right|+\left|c_{1}\right|^{2}+\left(1-\left|c_{0}\right|^{2}\right)^{2} \leqslant 1$ give the regions $B_{1}, B_{2}$ and $B_{3}$, respectively, according to Schur's algorithm. If we replace $c_{0}, c_{1}$ and $c_{2}$ in these inequalities by $c_{0}=\gamma_{0}, c_{1}=\gamma_{1}\left(1-|\zeta|^{2}\right)$ and $2 c_{2}=\gamma_{2}\left(1-|\zeta|^{2}\right)^{2}-2 \bar{\zeta} \gamma_{1}\left(1-|\zeta|^{2}\right)$, as implied by equation (10), we obtain the regions $B_{1}(\zeta), B_{2}(\zeta)$ and $B_{3}(\zeta)$. The regions $B_{n}(\zeta)$ may be more systematically determined by appealing to the general determinant inequalities which determine $B_{n}$ (see [10] or [11, p. 159]). The regions $S_{n}(\zeta)$ also may be expressed through equation (16) from the exact knowledge of the regions $B_{n}(\zeta)$ once $f$ is specified.

3. Except for the uniqueness statement in Theorem 4, the results in this section appear to hold in the more general setting of subordination, defined by equation (15), with the condition that $f$ is univalent in $\Delta$ replaced by $f$ is nonconstant. If $f$ is not univalent there no longer need be a one-to-one correspondence between boundary points of $S_{n}(f)$ and functions in $S(f)$. Even for $n=1$ this occurs, because whenever $f$ is not univalent on $\{z:|z|<r\}(0<r<1)$ there exist numbers $\zeta, x_{1}, x_{2}$ with $|\zeta|=r,\left|x_{1}\right|=\left|x_{2}\right|=1, x_{1} \neq x_{2}$ so that $f\left(x_{1} \zeta\right)=f\left(x_{2} \zeta\right)$.

If the univalence of $f$ is replaced by the condition that $f^{\prime}$ does not vanish, then equation (16) implies that (when $\zeta \neq 0$ ) an interior point of $B_{n}(\zeta)$ can be associated only with interior points of $S_{n}(\zeta)$. Namely, if $\left(w_{1}, w_{2}, \ldots, w_{n-1}\right)$ is determined by equation (17) from the interior point $\left(z_{1}, z_{2}, \ldots, z_{n-1}\right)$ then any point $\left(w_{1}^{\prime}\right.$, $\left.w_{2}^{\prime}, \ldots, w_{n-1}^{\prime}\right)$ near $\left(w_{1}, w_{2}, \ldots, w_{n-1}\right)$ also will be attained through these relations by successively determining $w_{1}^{\prime}, w_{2}^{\prime}, \ldots, w_{n-1}^{\prime}$. This uses the fact that $f\left(\zeta_{z}\right)$ is an open mapping and that once $z_{1}^{\prime}, z_{2}^{\prime}, \ldots, z_{k-1}^{\prime}$ are found $w_{k}^{\prime}$ may be obtained from a suitable value of $z_{k}^{\prime}$ through the linear correspondence between $z_{k}$ and $w_{k}$ (since $\alpha_{k} \neq 0$ ). This implies that Theorem 5 holds (when $\zeta \neq 0$ ) with the condition $f^{\prime}(z) \neq 0$ for $|z|<1$ replacing $f$ is univalent.

If $\zeta=0$ similar inferences can be made, In fact, simply if $a_{1}=f^{\prime}(0) \neq 0$ then equation (18) still provides a homeomorphism between $B_{n-2}$ and $S_{n}(0)$. It appears that a more general version of equations (16) and (18) (for example, where $a_{1} \neq 0$ is replaced by $a_{1}=a_{2}=\cdots=a_{m-1}=0$ and $a_{m} \neq 0$, in the case of equation (18)) will yield the results in Theorems 4 and 5 for general nonconstant functions $f$.

\section{REFERENCES}

1. A. Atzmon, Extremal functions for functionals on some classes of analytic functions, J. Math. Anal. Appl. 65 (1978), 333-338.

2. C. Carathéodory, Theory of functions of a complex variable, vol. 2, Chelsea, New York, 1954.

3. P. C. Cochrane and T. H. MacGregor, Fréchet differentiable functionals and support points for families of analytic functions, Trans. Amer. Math. Soc. 236 (1978), 75-92.

4. W. E. Kirwan, A note on extremal problems for certain classes of analytic functions, Proc. Amer. Math. Soc. 17 (1966), 1028-1030.

5. T. H. MacGregor, Applications of extreme-point theory to univalent functions, Michigan Math. J. 19 (1972), 361-376.

6. J. A. Pfaltzgraff, Extremal problems and coefficient regions for analytic functions represented by a Stieltjes integral, Trans. Amer. Math. Soc. 115 (1965), 270-282. 
7. M. S. Robertson, Extremal problems for analytic functions with pasitive real part and applications, Trans. Amer. Math. Soc. 106 (1963), 236-253.

8. K. Sakaguchi, A variational method for functions with positive real part, J. Math. Soc. Japan 16 (1964), 287-297.

9. J. Schur, Uber Potenzreihen, die im Innern des Einheitskreises beschränkt sind, J. Math. (Jabalpur) 147 (1917), 205-232.

10. _ Über Potenzreihen, die im Innern des Einheitskreises beschränkt sind, J. Math. (Jabalpur) 148 (1918), 122-145.

11. M. Tsuji, Potential theory in modern function theory, Maruzen, Tokyo, 1959.

Department of Mathematics, State Universtty of New York at Albany, Albany, New York 12222 